\title{
NIVELES DE HARINA DE ALGAS Nostoc commune EN EL INCREMENTO DE PESO VIVO EN CUYES (Cavia porcellus) DESTETADOS
}

\section{LEVELS OF FLOUR Nostoc commune ALGAE IN INCREASING IN GUINEA PIGS LIVE WEIGHT (Cavia porcellus) WEANED}

\author{
Carhuapoma D. V ${ }^{1^{*}}$, Valencia N. ${ }^{3}$, Mayhua, P. ${ }^{2}$, Sánchez A.V ${ }^{4}$ \\ ${ }^{1}$ Instituto de Investigación y Producción Científica de la Universidad Nacional de Huancavelica - \\ Huancavelica Perú. ${ }^{2}$ Ing. Zootecnista. Docente Asociado E.A.P. Zootecnia de la Universidad \\ Nacional de Huancavelica - Huancavelica Perú. ${ }^{3}$ Dr. Mvz. Docente principal E.A.P. Zootecnia de la \\ Universidad Nacional de Huancavelica - Huancavelica Perú. ${ }^{4}$ Dr. Mblgo. Docente principal E.A.P. \\ Zootecnia de la Universidad Nacional de Huancavelica - Huancavelica Perú \\ Autor para correspondencia: yachayruacc@hotmail.com
}

\section{RESUMEN}

El objetivo fue evaluar el Efecto de niveles de harina de Algas Nostoc commune en el incremento de peso vivo de cuyes destetados. Se evaluó en 64 cuyes de ambos sexos, de línea inti, con 20 días de edad. Los tratamiento consistieron en uso de diferentes niveles de harina de Nostoc commune como insumo proteico: T1 10\% harina de Nostoc commune y $90 \%$ de forraje de cebada, T2 15\% de harina de Nostoc Commune y $85 \%$ forraje de cebada, T3 20\% harina de Nostoc commune y $80 \%$ de forraje de cebada y T4 100\% forraje de cebada (testigo). Encontrando diferencia significativas de ganancia de peso, consumo de alimento y conversión alimenticia en el T2 en machos (2494,00 $\pm 2,26 \mathrm{~b}),(16.93 \pm 1.20 \mathrm{ab}),(3.9 \mathrm{~b})$ y hembras $(2410,00 \pm 2,07 \mathrm{~b})(17.29 \pm 1.22 \mathrm{ab}),(3.8 \mathrm{a})(\mathrm{P}<0.05)$, siendo inferiores $10 \mathrm{~s}$ 
tratamientos T1, T3 y T4. El nivel de15\% de harina de Nostoc commune fue óptimo para ganancia de peso de cuyes.

Palabras clave: Harina de Nostoc commune, cuyes, incremento de peso

\section{ABSTRACT}

The objective was to evaluate the effect of levels of algae Nostoc commune flour in live weight gain of weanling guinea pigs. 64 guinea pigs (males and females) inti line with 20 days of age were used. The treatment consisted of various levels using flour protein Nostoc commune as input : T1 10\% flour and $90 \%$ Nostoc commune fodder barley, T2 $15 \%$ flour and $85 \%$ Nostoc commune fodder barley , $20 \%$ T3 Nostoc commune flour and $80 \%$ forage barley and T4 $100 \%$ forage barley (control). Finding significant difference $(\mathrm{P}<0.01)$ body weight gain, feed intake and feed conversion in T2 in both sexes : males ( $2494.00 \pm 2,26 \mathrm{~b}$ ), $(16.93 \pm 1.20 \mathrm{ab}),(3.9 \mathrm{~b})$ and females $(2410.00 \pm 2,07 \mathrm{~b})(17.29 \pm 1.22 \mathrm{ab}),(3.8 \mathrm{a})(\mathrm{P}<$ 0.05), with lower T1, T3 and T4. The level of $15 \%$ flour Nostoc commune was optimal for increasing live weight of growing guinea pigs.

Key words: Nostoc meal, weight gain, guinea pigs green.

\section{INTRODUCCIÓN}

El consumo de la carne de cuyes en el Perú es limitado. Sin embargo, los cuyes son una especie potencialmente productora de carne, que aunado a su bajo costo de explotación, alta conversión alimenticia, gran prolificidad, rápido crecimiento, fácil manejo, añadiéndose a todo esto, la textura, suavidad y sabor de la carne que contienen de $22 \%$ de proteínas y con bajo \% de colesterol tiene demanda internacional, Castro (1998). Los altos costos de alimentos concentrados obligaron a los criadores a utilizar como insumos proteicos la harina de sangre, harina de huesos entre otros, que tampoco contemplan los requerimientos nutricionales de estos animales Escobar,(1998) debido a ello se propone utilizar con insumo proteico las algas de cianobacterias de las especies de N. commune, N. sphaericum, Spirulina platenses por presentar alto contenido de proteínas (35-42\%) y gran cantidad de clorofila y otros pigmentos como la C-ficocianina (Azul),C-ficoeritrina (Rojo), B-caroteno, aminoácidos esenciales y vitaminas, Zúñiga ( 1998), además las algas azul verdes se vino utilizando desde 
los tiempos muy remotas en la alimentación humana en forma directa como las especies de Nostoc (N. commune, N. sphaericum y N. pruniformae), que son denominados bajo los nombres vernáculares de "Cushuro", "Murmuta", "Llullusha"; y son consumidos en diversas localidades andinas del Perú, Ecuador y Bolivia, Dawson (1981). debido a ello, el objetivo del trabajo fue evaluar el efecto de diferentes niveles de harina de Nostoc commune como insumo proteico en el incremento de peso de cuyes, con la finalidad de abaratar los costos de producción e incrementar los parámetros productivos.

\section{MATERIAL Y MÉTODOS}

La investigación se llevó a cabo en la granja los "Andes" de la Comunidad Campesina de Antaccocha, ubicado a una altitud de $3800 \mathrm{msnm}$ con una temperatura media de $10^{\circ} \mathrm{C}$. Se utilizaron 24 jaulas modelo de madera con dimensiones de $60 * 50 * 40$, los cuales contaban con comederos, bebederos. Para este estudio se utilizaron 64 cuyes de ambos sexos (32 machos y 32 hembras) de línea inti, con 20 días de edad (destetados) y con un promedio de peso de $220 \mathrm{~g}$. El experimento tuvo una duración de 84 días. Las algas de cianobacterias de la especie Nostoc commune en estado natural se obtuvo de los riachuelos del Cerro de Huamanrrazu, que está ubicado en la Comunidad de Santa Bárbara - Huancavelica, épocas de invierno (meses de febrero y marzo), fueron deshidratados a temperatura ambiente bajo sombra / 18 días hasta obtener $2 \%$ de humedad), se removieron dos veces/día para la deshidratación uniforme y fueron procesados en harina, el análisis químico de la harina de Nostoc commune y Nostoc commune en estado natural fresco se realizó mediante el análisis Van Soes y el método Kjelldhal descrito por la Association of Official Analitical Chemists (AOAC, 1990) en el Laboratorio Central de Investigación de la Universidad Nacional de Huancavelica -1 Área de Alimentación Animal. Los tratamientos consistieron en el uso como insumo proteico la harina Nostoc commune, en los diferentes niveles: T1 $10 \%$ harina de Nostoc commune y $90 \%$ de forraje de cebada, T2 $15 \%$ de harina de Nostoc commune y $85 \%$ forraje de cebada, T3 $20 \%$ harina de Nostoc commune y 85 forraje de cebada y T4 100\% forraje de cebada como testigo. Las raciones de harina de Nostoc commune, afrecho de cebada, se formuló por el método de Cuadrado de Pearson simple, teniendo en cuenta el requerimiento nutricional de animal recomendada por Castro (2007). Las variables en estudio fueron: Incremento de peso total (IPT), consumo de alimento (COA) y la conversión alimenticia (CA). 


\section{RESULTADOS}

En la tabla 1, se aprecia que la harina de Nostoc commune presenta mayor contenido de proteínas $(32.40 \%)$, que en su estado natural fresco $(32 \%)$ y superior con respecto al forraje verde de cebada fresca (11.5\%).

Tabla 1: Análisis químico comparativo de Nostoc commune en estado natural, procesado y forraje verde de cebada.

\begin{tabular}{lcccc}
\hline \multirow{2}{*}{ NUTRIENTES } & \multirow{2}{*}{$\mathbf{N}^{\circ}$ DE } & \multicolumn{3}{c}{ TIPOS DE ALIMENTO } \\
& MUESTRAS & NEN & FV C & H.N \\
\hline PROTEINA TOTAL (\%) & 4 & 32,0 & 11,5 & 34,40 \\
EXTRACTO ETEREO (\%) & 4 & 4,7 & 3,2 & 4,2 \\
FIBRA CRUDA (\%) & 4 & 4,62 & $\mathbf{1 8 , 6 0}$ & 21,0 \\
HUMEDAD (\%) & 4 & 98,68 & $\mathbf{7 0 , 7}$ & $\mathbf{2 , 6 2}$ \\
MATERIA SECA (\%) & 4 & 1,32 & 29.30 & 97,38 \\
\hline
\end{tabular}

Leyenda: NEN $=$ Nostoc en estado natural fresco, $F V C=$ Forraje verde de cebada fresco, HN= Harina de Nostoc.

En base los resultados mostrados en la tabla 2, encontramos que el incremento de peso total y la conversión alimenticia fue significativo $(\mathrm{p}<0.05)$ con el tratamiento T2 en hembras $(2410,00 \pm 2,07 b) \mathrm{CA}(3.8 \mathrm{a})$ y en machos $(2494,00 \pm 2,26 \mathrm{~b}) \mathrm{CA}(3.9 \mathrm{~b})$, siendo inferiores los tratamientosT1, T3 y T4 (Testigo) tanto hembras y machos. Él mejor resultado del consumo de alimento se obtuvo con el tratamiento T1 (18.46 \pm 1.29 a) en machos y TI $(17.97 \pm 1.34$ a) para hembras, pero no influyeron en el incremento de pesos totales.

Tabla 2. Comparación de Medias, desviación Stardar de la ganancia de peso, consumo de alimentos y conversión alimenticia.

\begin{tabular}{|c|c|c|c|c|c|c|}
\hline \multirow[t]{2}{*}{ TRATA. } & \multicolumn{2}{|c|}{ INCREMENTO DE PESO TOTAL(G) } & \multicolumn{2}{|c|}{ CONSUMO DE ALIMENTO(KG) } & \multicolumn{2}{|c|}{$\begin{array}{l}\text { CONVERSION } \\
\text { ALIMENTICIA }\end{array}$} \\
\hline & Macho & Hembra & Macho & Hembra & $\mathbf{M}$ & $\mathbf{H}$ \\
\hline $\begin{array}{l}\text { T1 } 10 \% \\
\text { HN+ } \\
90 \% \text { FVC }\end{array}$ & $1992,42 \pm 2,75$ a & $1991,92 \pm 2,57 b$ & $17,97 \pm 1,34 b$ & $18,46 \pm 1,29$ a & $4,4 a$ & $5,3 \mathrm{~b}$ \\
\hline $\begin{array}{l}\text { T2 } 15 \% \\
\text { HN+ } 85 \% \\
\text { FVC }\end{array}$ & $2494,00 \pm 2,26 b$ & $2410,00 \pm 2,07$ a & $16,93 \pm 1,20$ a & $17,29 \pm 1,22 b$ & $3,9 b$ & $3,8 a$ \\
\hline $\begin{array}{l}\text { T3 } 20 \% \\
\text { HN+ } 80 \\
\text { FVC }\end{array}$ & $2028,83 \pm 3,91 \mathrm{a}$ & $2034,67 \pm 2,01 b$ & $17,21 \pm 1,20 \mathrm{a}$ & $18,22 \pm 1,26$ a & $4,9 \mathrm{~b}$ & $4,6 a$ \\
\hline $\begin{array}{l}\text { T4 } 100 \% \\
\text { de FVC }\end{array}$ & $1998,60 \pm 0,95 b$ & $1988,08 \pm 3,55 b$ & $13,15 \pm 1,07 b$ & $8,74 \pm 4,07 b$ & $5,2 a$ & $5,3 \mathrm{~b}$ \\
\hline
\end{tabular}


Letras iguales en la columna no tienen diferencias significativas $(p>0.05)$. H= Hembra, M= Macho.

\section{DISCUSIÓN}

En cuanto al valor hallado del porcentaje de proteína de $34 \%$ en la harina de Nostoc commune es superior con respecto al forraje verde de cebada (18.8\%) y en su estado natural (32\%), Chirinos (1998) relata valores que se encuentran entre 30 - 32\% de proteína, es inferior al que se obtuvo. Por otra Radwal et al. (2004), Reporta que Nostoc commune tiene $40-45 \%$ de proteínas, y la variedad Spirulina platenses entre $63-68 \%$ de proteína y todos los aminoácidos esenciales y vitaminas, nuestros resultados son inferiores debido a que la composición química de estas algas alto andinas varían debido a los cambios climatológico y de los componentes químicos de los recursos hídricos, Acleto (1998). En cuanto a los otros componentes químicos encontrados de Nostoc commune no se encontró ningún reporte para poder discutirlos.

El valor hallado del incremento de peso $(2410,00 \pm 2,07 b)$ y la conversión alimenticia (3.8a) fue significativo $(\mathrm{p}<0.05)$ con el tratamiento $\mathrm{T} 2$ en hembras y en machos, siendo inferiores los tratamientosT1, T3 y T4, en este estudio; siendo superiores a los resultados encontrados por Escobar et al.,( 1999),donde sus nos muestran una ganancias de peso de 693gr en machos y 630gr en hembras /animal con la inclusión de 10 y $15 \%$ de concentrado de harina de sangre, Mattos y Chauca (1999) quienes encontraron los datos inferiores a lo nuestro : incremento de peso total $\mathrm{T}-0 \approx 407.4 \mathrm{a}$ g, $\mathrm{T}-10 \approx 608.3 \mathrm{~b}$; $\mathrm{T}-20 \approx$ $647.5 \mathrm{~b}$ y $\mathrm{T}-30 \approx 674.8 \mathrm{~g}$, la conversión alimenticiaT-20 (4.68) y T-30 (4.70), T0 (6.15), y T-10 (5.05), utilizaron niveles de ensilado de pescado en la ración; esta diferencia que existe frente a los reportes comparados es debido a que las algas Nostoc commune contienen alto contenido de proteína y contempla satisfactoriamente su requerimiento nutricional del cuy.

Él mejor resultado del consumo de alimento se obtuvo con el tratamiento T1 (18.46 \pm 1.29 a) en machos y TI $(17.97 \pm 1.34$ a) para hembras, pero no influyeron en el incremento de pesos, debido a que los cuyes fisiológicamente no metabolizaron eficientemente los niveles bajos y altos de proteína en su ración proporcionado a lo contrario hacen desméritos en su parámetros productivos y reproductivos. 


\section{AGRADECIMIENTOS}

Al administrador ejecutivo del Laboratorio Central de Investigación de la Universidad Nacional de Huancavelica y la Granja los Andes de la comunidad de AntaccochaHuancavelica- Perú

\section{BIBLIOGRAFIA}

Acleto, C., Zuñiga, R. (1998) Introducción a las Algas Serie Didáctica de la Universidad Nacional Mayor de San Marcos. Pp -22-26

Castro, J. (1998) Suplementos con diferente nivel proteico en la Producción de carne de cuy bajo en grasa para consumo. XXII Reunión Científica Anual de la Asociación Peruana de Producción Animal Huancavelica- Perú .Pp 34-45

Castro, J. (1998) Transformación práctica de suplementos de inicio en suplementos de acabado para cuyes en engorde. XXII Reunión Científica Anual de la Asociación Peruana de Producción Animal Huancavelica. Perú .Pp 44-45

Chirinos, D., Espejos, J. (1994) Programa proteicos en las dietas izoenergéticas en la suplementación alimenticia de cuyes destetados. XXII Reunión Científica Anual de la Asociación Peruana de Producción Animal Huancavelica. Perú .Pp 22-32.

Escobar, F. (1998).Niveles de Harina de Sangre en Alimentación de Cuyes. XXII Reunión Científica Anual de la Asociación Peruana de Producción Animal Huancavelica. Perú .Pp 18-32.

Rivera, E., Castro, J. (1994) Efecto de la Adición de Dl-Metionina a Suplemento con Diferentes Niveles Proteicos para Cuyes en Crecimiento. XXII Reunión Científica Anual de la Asociación Peruana de Producción Animal Huancavelica-Perú. Perú .Pp 45-65 